\title{
Survey on Digital Watermarking Techniques
}

\author{
Deepti Shukla $^{1}$ and Nirupama Tiwari ${ }^{2}$ \\ ${ }^{1,2}$ Computer Science \& Engineering Dept., SRCEM, Gwalior \\ ${ }^{1}$ shukla.deepti12@gmail.com, ${ }^{2}$ girishniru@gmail.com
}

\begin{abstract}
This paper presents a literature survey on Digital Watermarking within an image. It describes the early work carried out on digital watermarks, including the brief analysis of various watermarking schemes and its applications. This paper also makes a comparison between various watermarking schemes. This paper also gives us a brief introduction about the procedure of digital watermarking.
\end{abstract}

Keywords: Digital Watermarking, DCT, DWT, DFT, LSB, Watermarked Image

\section{Introduction}

There are tons of data that is distributed over the internet. This data are stored and transmitted in a digital format and can easily be copied without loss of quality and efficiently distributed. That's why protection has become increasingly important.

Thus for hiding multimedia information, watermarking is a relative new technique. Its application is broad, including data authentication, protection of ownership, broadcast monitoring etc. Basically watermarking can easily be defined as the process of embedding watermarks in digital media e.g. audio, video, image etc. using an appropriate algorithm. The purpose of watermark is vast including the identification of work and discourages its unauthorized use etc. For an example, you may take a digital picture of an event that you may consider selling to a p. However, since you are greedy a human being, you might have sent the photos to bunch of different companies to make them go on a bidding war. One individual that works at company may then modify the image a bit, claim that it's their original work, and essentially steal it. So what are you left with? Nothing, unfortunately, because you did not know that you can protect your image even if it's in a digital format. How? You can embed extra information into digitized data to use as a protection - that is what digital watermarking is essentially. It is very similar to Steganography in a number of respects. The main aim of digital image watermarking is to embed information imperceptibly and robustly in the cover data. Basically digital watermarking is used for the security of the digital content and to protect the data from illegal use and provides the ownership right for the digital data. The efficiency of digital watermarking algorithms is totally based on the robustness of the embedded watermark against various types of attacks like cropping, low pass filter, Salt and Pepper noise and JPEG compression.

Digital watermarking is a very developing field and it is used in various applications most of which proved to be successful. The aim of every application is to provide security to the digital content.

This paper is categorized in different sections. In Section 2, we have discussed working of digital image watermarking with explanation. Section3 defines digital watermarking techniques and Section 4 describes the experimental results of some important spatial domain or transforms domain techniques. Finally, Section 5 draws conclusions. 


\section{Classification of Digital Watermarking}

There are many algorithms which are being used to hide the secret in-formation. These algorithms can be categorized into two domains called:
A. Spatial domain and

B. Frequency domain.

Spatial domain watermarking slightly modifies the pixels of one or two randomly selected subsets of an image. On the other side, in frequency domain techniques the image is first transformed to the frequency domain by the use of any transformation methods such as Fourier transform, discrete cosine transform (DCT) or discrete wavelet transform (DWT). Now the information is added to the values of its transform coefficients. After applying the inverse transform, the marked coefficients form the embedded image.

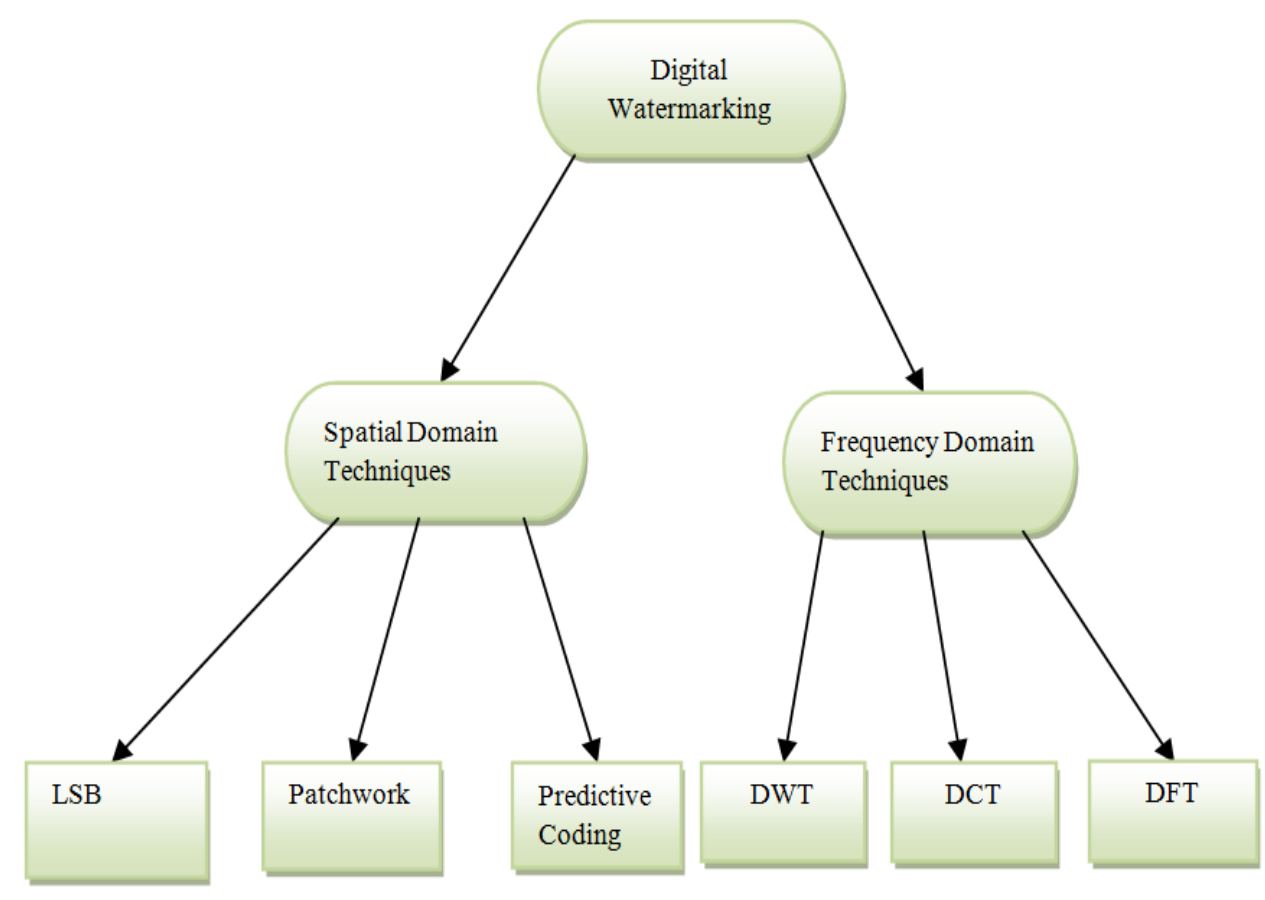

Figure 1. Classification of Digital Watermarking

\section{A. Spatial Domain}

\section{A.1. Least Significant bit (LSB)}

In this technique watermark is embedded in the LSB of pixels. Two types of LSB techniques are proposed. In the first method the LSB of the image was replaced with a pseudo-noise (PN) sequence while in the second a PN sequence was added to the LSB. This method is easy to use but not very robust against attacks.

\section{A.2. Patchwork Technique}

In patchwork, $n$ pairs of image points, (a,b),were randomly chosen. The image data in $a$ were lightened while that in $b$ were darkened .High level of robustness against many types of attacks are provided in this technique. But here in this technique, very small amount of information can be hidden. 


\section{A.3. Predictive Coding Scheme}

In this method, a pseudorandom noise (PN) pattern says $\mathrm{W}(\mathrm{x}, \mathrm{y})$ is added to cover image. It increases the robustness of watermark by increasing the gain factor. But due to high increment in gain factor, image quality may decrease.

\section{B. Frequency Domain}

\section{B.1. Discrete Cosine Transforms (DCT)}

First of all image is segmented into non overlapping blocks of 8x8.Then forward DCT is applied to each of these blocks. After that some block selection criteria is applied and then coefficient selection criteria is applied .Then watermark is embedded by modifying the selected coefficients and in the end inverse DCT transform is applied on each 8x8 block.

\section{B.2. Discrete wavelets transform (DWT)}

It is more frequently used due to its time/frequency characteristics. Here an image is passed through series of low pass and high pass filters which decompose the image into sub bands of different resolutions. Image is decomposed into four parts, one part is a low frequency of original image, the one bottom left is vertical details of the original image, the top right contains horizontal detail of the image, the bottom right block contains high frequency of original image. This technique uses wavelet filters to transform the image.

\section{B.3. Discrete Fourier Transforms (DFT)}

It transforms a continuous function into its frequency components. Discrete Fourier transform is scaling, rotation and translation invariant whereas the spatial domain DCT and DWT are not RST invariant. So DFT can be used to recover from various geometric attacks such as cropping.

\section{Literature Review}

This section introduces the techniques and methods currently available in the area of digital image watermarking. It presents a review of some influential work in the area of digital watermarking technique. A survey of the current research is presented as well as an analysis of the current techniques and methods available for digital image watermarking are also presented.

Nirupama et al [3] proposed an algorithm to protect digital data by embedding watermark that is encrypted by DES algorithm. Two level discrete wavelet transformation (DWT) is applied to the original image before apply watermarking in it.

In 2009, Mei Jiansheng et al. [8] introduce a discrete wavelet transform digital watermark algorithm based on human vision characters. In this technique, first of all watermark image is transformed by using DCT transformation. Then this watermark image is embedded into the high frequency band of wavelet transformation domain.

In 2009, S.S. Bedi et al. [16] exploit the characteristic of the Human Visual System to embed a robust and imperceptible watermark in transform domain using edge detection.

In 2010, Chih Chin Lai et al. [7] proposes a new technique in which the watermark is not embedded directly on the wavelet coefficients but rather than on the elements of singular values of the cover image's DWT.

In 2012, Manoj Ramaiya et al. [17] proposed a method that is created for fingerprint recognition based on minutiae.

In 2012, Navnidhi Chaturvedi et al. [10] have compared watermarking using DWT \& DWT-DCT method's performance analysis on basis of PSNR. 
In the same way, in 2012, 1 level Discrete wavelet transform for insertion and extraction of watermark in original image by using alpha blending was proposed.

After that in 2013, a novel technique was proposed to protect the copyright information by embedding a bitmap image as watermark into the frames of videos. In addition to this, some surveys were made on various image encryption and decryption techniques as well as various techniques for video watermarking were also proposed in 2013.

Next in 2014,a joint application compression, encryption and embedding techniques for implementing Steganography was proposed by Palak Mahajan .In this technique, For compression JPEG -LS Compression is used, similarly substitution technique is used for encryption and least significant bit insertion is used for embedding technique.

In the same way in 2014, another fragile watermarking scheme based on chaotic system has been proposed. Two dimensional Arnold's cat map has been used to improve the security of the proposed watermarking scheme. The proposed scheme provides high security, extracts watermark from the tampered image and also localizes the tampered area.

In the same year, Upasna Yadav et al. surveyed the various aspects of digital watermarking and its applications. In 2014, Seyed et al proposes that watermarking in the regions which is known as the edges of the image has better result than other regions .Genetic algorithm is also used for optimizing the different embedding strength coefficient.

\section{Watermarking Insertion and Extraction}

Encoding an identifying code into a digitized signal, that signal could be music, video, picture or other file is known as digital watermarking. OR It can be defined as the process of hiding digital information in a carrier signal.

The process of watermarking insertion is as follows: First of all a suitable image is selected as a cover image. Similarly a watermark is also selected. After this that watermark is inserted in this cover image with the help of a suitable watermark embedding algorithm .We discussed about various watermark embedding algorithm above in this paper. The result we obtain is the watermarked image.

Similarly purpose of watermark extraction process is to extract the watermark from the watermarked image .For achieving this the watermark embedding algorithm is applied in the reverse order so as to separate the watermark from watermarked image.

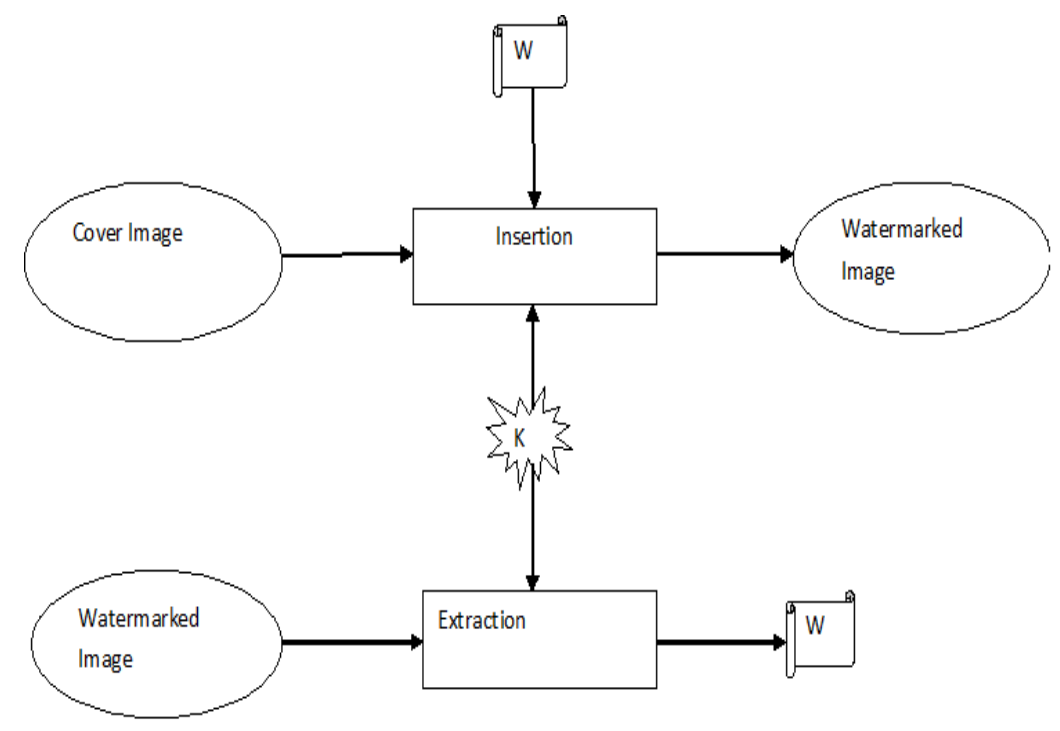

Figure 2. Insertion and Extraction of Watermarking 


\section{Applications of Digital Watermarking}

1. Owner Identification: It establishes ownership of the content.

2. Copy Protection: It prevent people from making illegal copies of copyright content.

3. Authentication of Content: To detect modifications of the content as a sign of invalid authentication.

4. Fingerprinting: Trace back illegal duplication and duplication of the content.

5. Broadcast Monitoring: Specially for advertisements and in entertainment industries, to monitor content that is broadcast as contracted and by the authorized source.

6. Medical Applications: Used to provide both authentication and confidentiality without affecting the medical image in any way.

\section{Conclusion}

This paper gives a detailed study on various digital watermarking techniques and their applications. It gives us a relative analysis of various watermarking techniques as well as general procedure of watermark embedding and extraction. In this paper, we tried our best to give the whole information of digital watermarking which will give benefit to new researchers to get the maximum awareness about this domain.

\section{References}

[1] Digital Image Processing by Rafael C. Gonzalez and Richard E. Woods.

[2] Digital Image Processing Using MATLAB by Rafael C. Gonzalez, Richard E. Woods and Steven L. Eddins.

[3] N. Tiwari, M. K. Ramaiya and M. Sharma, "Digital Watermarking using DWT and DES".

[4] P. Mahajan and A. Kohl, "CEET: A Compressed Encrypted \& Embedded Technique for Digital Image Steganography”, IOSR Journal Of Computer Engineering9IOSR-JCE) e-ISSN: 2278-0661,p-ISSN:22788727 vol. 16, no. 2, Ver. X (2014) March-April.

[5] Moniruzzaman, A. K. Hawlader and F. Hossai,"An Image Fragile Watermarking Scheme Based on Chaotic System for Image Tamper Detection", $3^{\text {rd }}$ International Conference on Informatics, Electronics \& Vision (2014).

[6] G. V. Mane* and G. G. Chiddarwar, "Review Paper on Video Watermarking Techniques", International Journal of Scientific and Research Publications, vol. 3, no. 4, (2013) April, 1 ISSN 2250-3153.

[7] C.-C. Lai and C.-C. Tsai, "Digital Image Watermarking Using Discrete Wavelet Transform and Singular Value Decomposition", IEEE Transactions on Instrumentation and Measurement, vol. 59, no. 11, (2010) November.

[8] J. Mei, S. Li and X. Tan, "A Digital Watermarking Algorithm Based on DCT and DWT”, IOSN 978-9525726-00-8, Proceedings of the 2009 International Symposium on Web Information Systems And Applications (WISA'09)Nanchang, P.R. China, (2009), May 22-24, pp. 104-107.

[9] G. S. Chandel and P. Patel, "A Review: Image Encryption with RSA and RGB Randomized Histograms", International Journal of Advanced Research In Computer And Communication Engineering, vol. 2, no. 11, (2013), November.

[10] M. Narang and S. Vashisth, "Digital Watermarking using Discrete Wavelet Transform", International Journal of Computer Applications (0975 - 8887) vol. 74, no. 20, (2013) July.

[11] N. Chaturvedi and S. J. Basha, "Comparison of Digital Image Watermarking methods DWT \& DWT$D C T$ on the basis of PSNR", International Journal Of Innovative Research in Science, Engineering and Technology, vol. 1, no. 2, (2012), December.

[12] S. S. M. Ziabari, R. E. Atani, K. Keyghobad and A. Riazi, "Digital Image watermarking using Edge Detection and Genetic Algorithm", International Journal of Scientific Engineering and Technology vol. 3 , no. 1.

[13] U. Yadav, J. P. Sharma, D. Sharma and P. K Sharma, "Different Watermarking Techniques And Its Applications: A Review", International Journal Of Scientific And Engineering Research, vol. 5, no. 4, (2014) April.

[14] Barun Pandhwal, D S Chaudhari “An Overview of Digital Watermarking Techniques" International Journal Of Soft Computing And Engineering (IJSCE) ISSN: 2231-2307, Volume-3, Issue-1, March 2013.

[15] S.S.Bedi, Rakesh CAhuja, Himanshu Agarwal "Copyright protection using video watermarking based on wavelet transformation in multiband". International journal of computer application (0975-8887) volume 66 no. 8 march 2013 . 
International Journal of Signal Processing, Image Processing and Pattern Recognition Vol.8, No.9 (2015)

[16] S.S. Bedi, G.S. Tomar, Shekhar Verma " Robust Watermarking of image in the transform domain using edge detection" UKSim 2009; $11^{\text {th }}$ international conference on computer modeling and simulation .

[17] Prof. Manoj Ramaiya "Fingerprint Recognition based on Minutiae Extraction Principle". 